\title{
Note sur les polynômes de Kazhdan-Lusztig
}

\author{
Sophie Morel
}

25 mars 2022

\begin{abstract}
Dans son article [B], Brenti a prouvé de manière combinatoire une formule non-récursive pour les polynômes de Kazhdan-Lusztig d'un groupe de Coxeter (cf le théorème 4.1 de [B]). Le but de cette note est de donner une interprétation géométrique de cette formule pour les groupes de Coxeter qui sont isomorphes à un groupe de Weyl. Cette interprétation utilise un résultat de $[\mathrm{M}]$ (théorème 3.3.5), qui exprime le prolongement intermédiaire d'un faisceau pervers pur comme "tronqué par le poids" de son image.

Donnons rapidement le plan de l'article. La section 1 rappelle la définition, due à Kazhdan et Lusztig, des polynômes de Kazhdan-Lusztig et des polynômes $R$. La section 2 rappelle le résultat de $[\mathrm{M}]$ qui sera utilisé dans l'interprétation géométrique de la formule de Brenti. La section 3 rappelle une décomposition des cellules de Bruhat due à Deodhar, et en donne une nouvelle preuve, qui utilise des calculs de Härterich. Cette décomposition sera utile dans la section 4, pour montrer que la cohomologie des cellules de Bruhat a une forme particulièrement simple. Enfin, la section 4 contient le résultat principal (théorème 4.1), c'est-à-dire la preuve géométrique de la formule de Brenti.

Je remercie Gérard Laumon de m'avoir signalé cette application de [M].
\end{abstract}

Dans toute la suite, $\mathbb{F}_{q}$ est un corps fini, $\mathbb{F}_{q} \subset \overline{\mathbb{F}}_{q}$ est une clôture algébrique de $\mathbb{F}_{q}$ et $\ell$ est un nombre premier inversible dans $\mathbb{F}_{q}$.

\section{Polynômes de Kazhdan-Lusztig et polynômes $R$}

Dans cette section, nous rappelons quelques résultats de Kazhdan et Lusztig (cf [KL1] et [KL2]).

Soit $G$ un groupe algébrique réductif connexe déployé sur $\mathbb{F}_{q}$. Soient $B$ un sous-groupe de Borel de $G$ (défini sur $\mathbb{F}_{q}$ ), $T \subset B$ un tore maximal déployé sur $\mathbb{F}_{q}, B^{*}$ le sous-groupe de Borel de $G$ opposé à $B$ (ie tel que $B \cap B^{*}=T$ ), $N$ le normalisateur de $T$ dans $G$ et $W=N / T$ le groupe de Weyl. On note $\Phi$ l'ensemble des racines de $T$ dans $\operatorname{Lie}(G), \Phi^{+}$le sous-ensemble de racines positives associé à $B$ (c'est-à-dire l'ensemble des racines de $T$ dans $\operatorname{Lie}(B)$ ), et $\Delta \subset \Phi^{+}$l'ensemble des racines simples. Enfin, on note $\ell$ la fonction longueur sur $W$ associée à $\Delta$ et $\leqslant$ l'ordre de Bruhat sur $W$. 
Rappelons que l'algèbre de Hecke $\mathcal{H}$ de $W$ est l'algèbre sur l'anneau $\mathbb{Z}\left[t^{1 / 2}, t^{-1 / 2}\right]$ engendrée par des éléments $T_{w}, w \in W$, soumis aux relations :

$$
\begin{array}{ll}
T_{w} T_{w^{\prime}}=T_{w w^{\prime}} & \text { si } \ell\left(w w^{\prime}\right)=\ell(w)+\ell\left(w^{\prime}\right) \\
\left(T_{s}+1\right)\left(T_{s}-t\right)=0 & \text { si } s \text { est la réflexion associée à une racine simple. }
\end{array}
$$

On définit des polynômes $R_{v, w} \in \mathbb{Z}[t], v, w \in W, v \leqslant w$, par les formules (cf [KL1] $\S 2$ ) :

$$
T_{w}^{-1}=\sum_{v \leqslant w}(-1)^{\ell(w)-\ell(v)} R_{v, w}(t) t^{-\ell(w)} T_{v} .
$$

$R_{v, w}$ est de degré $\ell(w)-\ell(v)$.

D'après le théorème 1.1 de KL1] (cf aussi KL2] $\S 2$ ), il existe une unique famille de polynômes $P_{v, w} \in \mathbb{Z}[t], v, w \in W, v \leqslant w$, avec $P_{w, w}=1$ et $P_{v, w}$ de degré $\leqslant \frac{1}{2}(\ell(w)-\ell(v)-1)$ si $v<w$, telle que : pour tous $v, w \in W$ tels que $v \leqslant w$,

$$
t^{\ell(w)-\ell(v)} P_{v, w}\left(t^{-1}\right)=\sum_{v \leqslant y \leqslant w} R_{v, y}(t) P_{y, w}(t) .
$$

Rappelons l'interprétation géométrique des polynômes $P_{v, w}$ donnée par Kazhdan et Lusztig dans [KL2].

Le choix de $B$ détermine un isomorphisme entre la variété $\mathcal{B}$ des sous-groupes de Borel de $G$ et le quotient $G / B$. On a deux stratifications de $\mathcal{B}$ par des sous-variétés localement fermées, $\mathcal{B}=\bigcup_{w \in W} X_{w}=\bigcup_{w \in W} X^{w}$, avec, pour tout $w \in W$,

$$
\begin{gathered}
X_{w}=\left\{B^{\prime} \in \mathcal{B} \mid \exists g \in B w B, B^{\prime}=g B g^{-1}\right\} \\
X^{w}=\left\{B^{\prime} \in \mathcal{B} \mid \exists g \in B^{*} w B, B^{\prime}=g B g^{-1}\right\} .
\end{gathered}
$$

La variété $X_{w}$ est isomorphe à l'espace affine $\mathbb{A}^{\ell(w)}$, et $X^{w}$ est isomorphe à $\mathbb{A}^{\operatorname{dim}(\mathcal{B})-\ell(w)}$. Pour tout $w \in W$, on note $x_{w}=w B w^{-1} \in X_{w} \cap X^{w}$.

L'adhérence $\bar{X}_{w}$ de $X_{w}$ dans $\mathcal{B}$ est la variété de Schubert associée à $w$. C'est une variété projective irréductible de dimension $\ell(w)$, et on a

$$
\bar{X}_{w}=\bigcup_{v \leqslant w} X_{v} .
$$

De même, on a

$$
\bar{X}^{w}=\bigcup_{v \geqslant w} X^{v}
$$

On note $F: \mathcal{B} \longrightarrow \mathcal{B}$ le Frobenius. Pour tout $w \in W$, on a $F\left(X_{w}\right) \subset F\left(X_{w}\right), F\left(X^{w}\right) \subset$ $F\left(X^{w}\right)$ et $F\left(x_{w}\right)=x_{w}$. Pour tous $v, w \in W$ tels que $v \leqslant w$, on note $j_{w}$ et $i_{v, w}$ les inclusions de $X_{w}$ et $X_{v}$ dans $\bar{X}_{w}$, et

$$
I C_{\bar{X}_{w}}=\left(j_{w ! *}\left(\mathbb{Q}_{\ell}[\ell(w)]\right)\right)[-\ell(w)]
$$

le complexe d'intersection à coefficients constants de $\bar{X}_{w}$.

Kazhdan et Lusztig ont démontré le théorème suivant ([KL2], théorèmes 4.2 et 4.3) : 
Théorème 1.1 (Kazhdan-Lusztig) Soit $w \in W$. Alors le faisceau de cohomologie (ordinaire) $\mathcal{H}^{i}\left(I C_{\bar{X}_{w}}\right)$ est nul si $i$ est impair. Si $i$ est pair et $B^{\prime} \in \bar{X}_{w}$ est stable par une puissance $F^{r}$ de $F$, alors les valeurs propres de $\left(F^{r}\right)^{*}$ sur la fibre $\mathcal{H}^{i}\left(I C_{\bar{X}_{w}}\right)_{B^{\prime}}$ sont toutes égales à $q^{\text {ir } / 2}$.

De plus, pour tout $v \leqslant w$, on a

$$
P_{v, w}(t)=\sum_{i \geqslant 0} \operatorname{dim}\left(\mathcal{H}^{2 i}\left(I C_{\bar{X}_{w}}\right)_{x_{v}}\right) t^{i}
$$

\section{Rappel d'un résultat de [M]}

Soit $X$ un schéma séparé de type fini sur $\mathbb{F}_{q}$. On note $D_{m}^{b}\left(X, \mathbb{Q}_{\ell}\right)$ la catégorie des complexes $\ell$-adiques mixtes sur $\mathbb{F}_{q}$. Cette catégorie est munie de la t-structure donnée par la perversité autoduale, et on note ${ }^{p} \mathrm{H}^{i}$ les foncteurs de cohomologie pour cette t-structure. (Les notions de ce paragraphe sont définies dans le chapitre 5 du livre [BBD] de Beilinson, Bernstein et Deligne.)

Pour tout $a \in \mathbb{Z} \cup\{ \pm \infty\}$, on note ${ }^{w} \mathrm{D}^{\leqslant a}(X)$ (resp. ${ }^{w} \mathrm{D}^{\geqslant a}(X)$ ) la sous-catégorie pleine de $D_{m}^{b}\left(X, \mathbb{Q}_{\ell}\right)$ dont les objets sont les complexes mixtes $K$ tels que pour tout $i \in \mathbb{Z}$, le faisceau pervers ${ }^{p} \mathrm{H}^{i} K$ soit de poids $\leqslant a($ resp. $\geqslant a)$. Alors $([\mathbb{M}]$, proposition 3.1.1 (iv)) :

Proposition 2.1 Pour tout $a \in \mathbb{Z} \cup\{ \pm \infty\},\left({ }^{w} \mathrm{D}^{\leqslant a}(X),{ }^{w} \mathrm{D}^{\geqslant a+1}(X)\right)$ est une t-structure sur $D_{m}^{b}\left(X, \mathbb{Q}_{\ell}\right)$.

On note $w_{\leqslant a}$ et $w_{\geqslant a+1}=w_{>a}$ les foncteurs de troncature pour cette t-structure. Ce sont des foncteurs triangulés ( $\operatorname{car}^{w} \mathrm{D}^{\leqslant a}(X)$ et ${ }^{w} \mathrm{D}^{\geqslant a+1}(X)$ sont des sous-catégories triangulées de $\left.D_{m}^{b}\left(X, \mathbb{Q}_{\ell}\right)\right)$, et la dualité de Poincaré échange $w_{\leqslant a}$ et $w_{\geqslant-a}$.

Soit $\left(S_{0}, \ldots, S_{n}\right)$ une partition de $X$ par des sous-schémas (localement fermés non vides) telle que, pour tout $k \in\{0, \ldots, n\}, S_{k}$ soit ouvert dans $X-\bigcup_{l<k} S_{l}$. En particulier, $S_{0}$ est ouvert dans $X$. Pour tout $k \in\{0, \ldots, n\}$, on note $i_{k}$ l'inclusion de $S_{k}$ dans $X$.

Le théorème suivant découle des résultats de la section 3 de $[\mathrm{M}]$ :

Théorème 2.2 Soient $a \in \mathbb{Z}$ et $K$ un faisceau pervers pur de poids a sur $S_{0}$. Alors on a une égalité de classes dans le groupe de Grothendieck de $D_{m}^{b}\left(X, \mathbb{Q}_{\ell}\right)$ :

$$
\begin{aligned}
{\left[i_{0 ! *} K\right]=} & \sum_{1 \leqslant n_{1}<\cdots<n_{r}<n}(-1)^{r}\left[i_{n_{r} !} w_{\leqslant a} i_{n_{r}} \ldots i_{n_{1} !} w_{\leqslant} i_{n_{1}} i_{0 !} K\right] \\
& +\sum_{1 \leqslant n_{1}<\cdots<n_{r}=n}(-1)^{r}\left[i_{n_{r} !} w_{<a} i_{n_{r}} i_{n_{r-1} !} w_{\leqslant a} i_{n_{r-1}}^{!} \ldots i_{n_{1} !} w_{\leqslant a} i_{n_{1}} i_{0 !} K\right] .
\end{aligned}
$$

Démonstration. On utilise les notations de la section 3 de [M]. D'après la proposition [M] 3.4 .2 , on a des isomorphismes canoniques

$$
w_{\geqslant(a, a+1, \ldots, a+1)} i_{0 !} K=w_{\geqslant(a, a, \ldots, a)} i_{0 !} K=i_{0 ! *} K,
$$


d'où un isomorphisme canonique

$$
w_{\geqslant(a, a+1, \ldots, a+1, a)} i_{0 !} K=i_{0 ! *} K .
$$

De plus, le théorème $[\mathrm{M}] 3.3 .5$ donne pour tout $\underline{a}=\left(a_{0}, \ldots, a_{n}\right) \in(\mathbb{Z} \cup\{ \pm \infty\})^{n+1}$ tel que $a=a_{0}$ une égalité dans le groupe de Grothendieck de $D_{m}^{b}\left(X, \mathbb{Q}_{\ell}\right)$ :

$$
\left[w_{\leqslant \underline{a}} R i_{0 *} K\right]=\sum_{1 \leqslant n_{1}<\cdots<n_{r} \leqslant n}(-1)^{r}\left[R i_{n_{r} *} w_{>a_{n_{r}}} i_{n_{r}}^{*} \ldots R i_{n_{1} *} w_{>a_{n_{1}}} i_{n_{1}}^{*} R i_{0 *} K\right],
$$

d'où par dualité :

$$
\left[w_{\geqslant a} i_{0 !} K\right]=\sum_{1 \leqslant n_{1}<\cdots<n_{r} \leqslant n}(-1)^{r}\left[i_{n_{r} !} w_{<a_{n_{r}}} i_{n_{r}}^{!} \ldots i_{n_{1} !} w_{<a_{n_{1}}} i_{n_{1}}^{!} i_{0 !} K\right] .
$$

Cette égalité, combinée avec l'isomorphisme ci-dessus, donne le résultat cherché.

\section{Décomposition des $X_{w}$}

Dans [D2], Deodhar a construit une décomposition des cellules de Bruhat $X_{w}$ qui induit une décomposition agréable des $X_{w} \cap X^{v}$, pour $v \leqslant w$. Nous allons donner ici une autre interprétation de cette décomposition.

Soit $w \in W$. On fixe une décomposition $w=s_{1} \ldots s_{r}$ de $w$ en réflexions simples, avec $r=\ell(w)$. Pour tout $i \in\{1, \ldots, r\}$, on note $\alpha_{i}$ la racine simple correspondant à $s_{i}$. Soit $\Gamma=\left\{1, s_{1}\right\} \times \cdots \times\left\{1, s_{r}\right\}$. Pour tout $\gamma=\left(\gamma_{1}, \ldots, \gamma_{r}\right) \in \Gamma$, on note

$$
\begin{gathered}
I(\gamma)=\left\{i \in\{1, \ldots, r\} \mid \gamma_{i}=s_{i}\right\} \\
J(\gamma)=\left\{i \in\{1, \ldots, r\} \mid \gamma_{1} \gamma_{2} \ldots \gamma_{i}\left(-\alpha_{i}\right) \in \Phi^{+}\right\} .
\end{gathered}
$$

Pour tout $v \leqslant w$, on note

$$
\Gamma_{v}=\left\{\left(\gamma_{1}, \ldots, \gamma_{r}\right) \in \Gamma \mid \gamma_{1} \ldots \gamma_{r}=v\right\} .
$$

Deodhar a montré le résultat suivant ([D2], théorème 1.1 et corollaire 1.2) :

Proposition 3.1 (Deodhar) La cellule de Bruhat $X_{w}$ s'écrit de manière canonique comme une union disjointe de sous-variétés localement fermées

$$
X_{w}=\bigcup_{\gamma \in \Gamma} Y_{\gamma},
$$

avec $Y_{\gamma}=\varnothing$ si $J(\gamma) \not \subset I(\gamma)$, et, pour tout $\gamma \in \Gamma$ tel que $J(\gamma) \subset I(\gamma)$,

$$
Y_{\gamma} \simeq \mathbb{A}^{\operatorname{card}(I(\gamma)-J(\gamma))} \times \mathbb{G}_{m}^{r-\operatorname{card}(I(\gamma))} .
$$

De plus, pour tout $v \in W$ tel que $v \leqslant w$, on a

$$
X_{w} \cap X^{v}=\bigcup_{\gamma \in \Gamma_{v}} Y_{\gamma} .
$$


En utilisant des calculs de Härterich $([\mathrm{H}], \S 1)$, on peut donner une autre preuve de ce résultat:

Soit $\lambda: \mathbb{G}_{m} \longrightarrow T$ un cocaractère tel que $\langle\alpha, \lambda\rangle>0$ pour tout $\alpha \in \Phi^{+}$. On fait agir $\mathbb{G}_{m}$ $\operatorname{sur} \mathcal{B}$ via $\lambda$. Les points fixes de cette action sont les $x_{z}, z \in W$, et les deux décompositions de Bialynicki-Birula (cf $[\mathrm{BB}]$ ) de $\mathcal{B}$ sont :

- la décomposition en cellules contractantes, $\mathcal{B}=\bigcup_{z \in W} X_{z}$;

- la décomposition en cellules dilatantes, $\mathcal{B}=\bigcup_{z \in W} X^{z}$.

On va décomposer $X_{w}$ en utilisant la décomposition de Bialynicki-Birula de la résolution de Bott-Samelson de $\bar{X}_{w}$ associée à la décomposition $w=s_{1} \ldots s_{r}$.

Pour toute racine $\alpha$, on note $p_{\alpha}: \mathbb{A}^{1} \longrightarrow U_{\alpha}$ le sous-groupe à un paramètre associé à $\alpha$. Si $\alpha$ est une racine simple, on note $P_{\alpha}$ le sous-groupe parabolique (minimal) de $G$ engendré par $B$ et $\operatorname{par} U_{-\alpha}$.

Dans [D1], Demazure a associé à la décomposition $w=s_{1} \ldots s_{r}$ de $w$ une résolution des singularités $\pi: B S \longrightarrow \bar{X}_{w}$ (appelée résolution de Bott-Samelson), où $B S=P_{\alpha_{1}} \times_{B} \cdots \times \times_{B}$ $P_{\alpha_{r}} / B$ et $\pi$ envoie la classe $\left[p_{1}, \ldots, p_{r}\right]$ de $\left(p_{1}, \ldots, p_{r}\right) \in P_{\alpha_{1}} \times \cdots \times P_{\alpha_{r}}$ dans $B S$ sur la classe de $p_{1} \ldots p_{r}$ dans $\bar{X}_{w} \simeq \overline{B w B} / B$. La restriction de $\pi$ à $\pi^{-1}\left(X_{w}\right)$ induit un isomorphisme $\pi^{-1}\left(X_{w}\right) \longrightarrow X_{w}$.

On fait agir $B$ sur $B S$ par multiplication à gauche sur le premier facteur. (Le morphisme $\pi$ est alors $B$-équivariant.) En particulier, on a une action de $\mathbb{G}_{m}$ sur $B S$ (via le cocaractère $\left.\lambda: \mathbb{G}_{m} \longrightarrow T\right)$, dont les points fixes sont les $\left[\gamma_{1}, \ldots, \gamma_{r}\right],\left(\gamma_{1}, \ldots, \gamma_{r}\right) \in \Gamma$. Dans $[\mathrm{H}]$, Härterich a décrit la décomposition en cellules contractantes de $B S$. Nous allons rappeler ses résultats.

Pour tout $\left(\gamma_{1}, \ldots, \gamma_{r}\right) \in \Gamma$, le morphisme $u_{\gamma}=\left(p_{\gamma_{1}\left(-\alpha_{1}\right)}, \ldots, p_{\gamma_{r}\left(-\alpha_{r}\right)}\right): \mathbb{A}^{r} \longrightarrow B S$ est une immersion ouverte $(\mathrm{cf}[\mathrm{H}], \S 1)$. On note $U_{\gamma}$ son image. On a $U_{\left(s_{1}, \ldots, s_{r}\right)}=\pi^{-1}\left(X_{w}\right)$ et, pour tout $\gamma=\left(\gamma_{1}, \ldots, \gamma_{r}\right) \in \Gamma, U_{\gamma} \cap U_{\left(s_{1}, \ldots, s_{r}\right)}=u_{\gamma}\left(\left\{\left(x_{1}, \ldots, x_{r}\right) \in \mathbb{A}^{r}, x_{i} \neq 0\right.\right.$ si $\left.\gamma_{i}=1\right\}$.

Soit $\gamma=\left(\gamma_{1}, \ldots, \gamma_{r}\right) \in \Gamma$. Härterich $([\mathbf{H}]$, formule 1.3) montre que la cellule contractante associée à $\left[\gamma_{1}, \ldots, \gamma_{r}\right]$ est

$$
C_{\gamma}=u_{\gamma}\left(\left\{\left(x_{1}, \ldots, x_{r}\right) \in \mathbb{A}^{r}, x_{i}=0 \text { si } i \notin J(\gamma)\right\}\right) .
$$

La même méthode permet de montrer que la cellule dilatante associée à $\gamma$ est

$$
C^{\gamma}=u_{\gamma}\left(\left\{\left(x_{1}, \ldots, x_{r}\right) \in \mathbb{A}^{r}, x_{i}=0 \text { si } i \in J(\gamma)\right\}\right) .
$$

La variété $B S$ est union disjointe des sous-variétés localement fermées $C^{\gamma}, \gamma \in \Gamma$, et le morphisme $\pi: B S \longrightarrow \bar{X}_{w}$ est un isomorphisme au-dessus de $X_{w}$, donc on a

$$
X_{w} \simeq \bigcup_{\gamma \in \Gamma}^{\bullet} U_{\left(s_{1}, \ldots, s_{r}\right)} \cap C^{\gamma} .
$$

Pour tout $\gamma \in \Gamma$, on pose

$$
Y_{\gamma}=U_{\left(s_{1}, \ldots, s_{r}\right)} \cap C^{\gamma} .
$$

Soit $\gamma \in \Gamma$. D'après les formules ci-dessus pour $C^{\gamma}$ et $U_{\left(s_{1}, \ldots, s_{r}\right)} \cap U_{\gamma}$, on a

$$
Y_{\gamma}=u_{\gamma}\left(\left\{\left(x_{1}, \ldots, x_{r}\right) \in \mathbb{A}^{r}, x_{i} \neq 0 \text { si } i \notin I(\gamma) \text { et } x_{i}=0 \text { si } i \in J(\gamma)\right\}\right) \text {. }
$$


Donc $Y_{\gamma}=\varnothing$ si $J(\gamma) \not \subset I(\gamma)$ et, si $J(\gamma) \subset I(\gamma)$,

$$
Y_{\gamma} \simeq \mathbb{A}^{\operatorname{card}(I(\gamma)-J(\gamma))} \times \mathbb{G}_{m}^{r-\operatorname{card}(I(\gamma))} .
$$

Enfin, comme le morphisme $\pi$ est $T$-équivariant, on a

$$
\pi^{-1}\left(\bar{X}_{w} \cap X^{v}\right)=\bigcup_{\gamma \in \Gamma_{v}} C^{\gamma}
$$

d'où la deuxième formule de la proposition.

\section{Calcul des polynômes de Kazhdan-Lusztig en fonction des polynômes $R$}

Pour tout $d \in \mathbb{Q}$, on définit un endomorphisme $\mathbb{Q}$-linéaire $\tau_{\leqslant d}$ de l'anneau des polynômes de Laurent $\mathbb{Q}\left[t^{1 / 2}, t^{-1 / 2}\right]$ par :

$$
\tau_{\leqslant d}\left(\sum_{i \in \mathbb{Z}} a_{i} t^{i / 2}\right)=\sum_{i \leqslant d} a_{i} t^{i / 2} .
$$

Le but de cette section est de donner une preuve géométrique du résultat suivant, qui a été prouvé de manière combinatoire par Brenti (cf [B], théorème 4.1) :

Théorème 4.1 Avec les notations de la section 1 , pour tous $v, w \in W$ tels que $v \leqslant w$, le polynôme $P_{v, w}$ est égal à :

$$
\begin{gathered}
\tau_{\leqslant \ell(w)-\ell(v)-1}\left(\sum_{v=v_{1}<\cdots<v_{r}<w}(-1)^{r} R_{v_{1}, v_{2}} \tau_{\leqslant \ell}(w)-\ell\left(v_{2}\right)\right. \\
\ldots \tau_{\leqslant \ell(w)-\ell\left(v_{r-1}\right)}\left(R_{v_{2}, v_{3}} \ldots\right. \\
\left.\left.\left.\ldots R_{v_{r-1}, v_{r}} \tau_{\leqslant \ell(w)-\ell\left(v_{r}\right)}\left(R_{v_{r}, w}\right)\right) \ldots\right)\right) .
\end{gathered}
$$

Pour tout schéma lisse $X$ sur $\mathbb{F}_{q}$, notons $\mathcal{T}(X)$ la sous-catégorie triangulée de $D_{m}^{b}\left(X, \mathbb{Q}_{\ell}\right)$ engendrée par les objets isomorphes aux faisceaux $\mathbb{Q}_{\ell}(m), m \in \mathbb{Z}$. Le groupe de Grothendieck $K(\mathcal{T}(X))$ de $\mathcal{T}(X)$ est le groupe abélien libre engendré par les $\left[\mathbb{Q}_{\ell}(m)\right], m \in \mathbb{Z}$. On définit un isomorphisme de groupes $\varphi: \mathcal{T}(X) \longrightarrow \mathbb{Z}\left[t, t^{-1}\right]$ par $\varphi\left(\left[\mathbb{Q}_{\ell}(m)\right]\right)=t^{-m}$, pour tout $m \in \mathbb{Z}$. Si $K$ est un objet de $\mathcal{T}(X)$ et $j \in \mathbb{N}^{*}$, on a, pour tout $x \in X\left(\mathbb{F}_{q^{j}}\right)$,

$$
\varphi([K])\left(q^{j}\right)=\operatorname{Tr}\left(F^{j *}, K_{x}\right) .
$$

Lemme 4.2 (i) Soit $X$ lisse connexe sur $\mathbb{F}_{q}$. Pour tout objet $K$ de $\mathcal{T}(X)$ et tout $a \in \mathbb{Z}$, on $a$ :

$$
\varphi\left(\left[w_{\leqslant a} K\right]\right)=\tau_{\leqslant a-\operatorname{dim}(X)}(\varphi([K])) .
$$


(ii) Pour tous $v, w \in W$ tels que $v \leqslant w$ et tout $K \in \mathcal{T}\left(X_{w}\right)$, le complexe $i_{v, w}^{!} j_{w !} K$ est dans $\mathcal{T}\left(X_{v}\right)$, on a un isomorphisme $\operatorname{Gal}\left(\overline{\mathbb{F}}_{q} / \mathbb{F}_{q}\right)$-équivariant

$$
\left(i_{v, w}^{!} j_{w !} K\right)_{x_{v}} \simeq R \Gamma_{c}\left(\left(X_{w} \cap X^{v}\right)_{\overline{\mathbb{F}}_{q}}, K_{\mid X_{w} \cap X_{v}}\right)
$$

et $\varphi\left(\left[i_{v, w}^{!} j_{w !} K\right]\right)=\varphi([K]) R_{v, w}(t)$.

(iii) Pour tous $v, w \in W$ tels que $v \leqslant w$, le complexe $i_{v, w}^{*} I C_{\bar{X}_{w}}$ est dans $\mathcal{T}\left(X_{v}\right)$.

Démonstration.

(i) On peut supposer que $K=\mathbb{Q}_{\ell}(m)$. L'égalité est alors évidente.

(ii) On peut supposer que $K=\mathbb{Q}_{\ell}(m)$. Le complexe $i_{v, w}^{!} j_{w}$ ! $K$ est un complexe $B$-équivariant sur $X_{v}$. Comme $B$ agit transitivement sur $X_{v}$ et que les stabilisateurs des points de $X_{v}$ sont connexes, les faisceaux de cohomologie de $i_{v, w}^{!} j_{w !} K$ sont constants. Il suffit donc de montrer que $\left(i_{v, w}^{!} j_{w !} K\right)_{x_{v}} \in \mathcal{T}\left(x_{v}\right)$.

D'après [KL2] 1.4, on a un diagramme commutatif $T$-équivariant

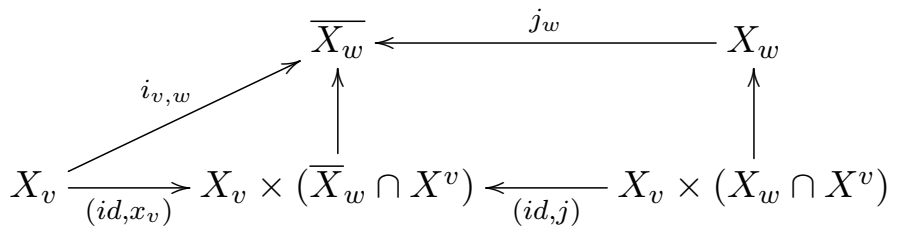

où les flèches verticales sont des immersions ouvertes et $j$ est l'inclusion $X_{w} \cap X^{v} \longrightarrow$ $\bar{X}_{w} \cap X^{v}$. D'après la formule de Künneth (cf SGA 5 III 1.7), on a

$$
i_{v, w}^{!} j_{w !} K=\left(i d, x_{v}\right)^{!}(i d, j)_{!}\left(\mathbb{Q}_{\ell, X_{v}} \otimes \mathbb{Q}_{\ell, X_{w} \cap X^{v}}(m)\right)=\mathbb{Q}_{\ell, X_{v}} \otimes\left(x_{v}^{!} j_{!} \mathbb{Q}_{\ell}(m)\right),
$$

donc

$$
\left(i_{v, w}^{!} j_{w !} K\right)_{x_{v}}=x_{v}^{!} j_{!} \mathbb{Q}_{\ell}(m)=x_{v}^{!} j_{w !}\left(K_{\mid X_{w} \cap X^{v}}\right) .
$$

D'après le sous-lemme 1] (qu'on peut appliquer par [KL2] 1.5), on a un isomorphisme $\operatorname{Gal}\left(\overline{\mathbb{F}}_{q} / \mathbb{F}_{q}\right)$-équivariant

$$
\left(i_{v, w}^{!} j_{w !} K\right)_{x_{v}}=x_{v}^{!} j_{!}\left(K_{\mid X_{w} \cap X^{v}}\right) \simeq R \Gamma_{c}\left(\left(X_{w} \cap X^{v}\right)_{\overline{\mathbb{F}}_{q}}, K_{\mid X_{w} \cap X^{v}}\right) .
$$

De plus, $X_{w} \cap X^{v}$ est union disjointe de schémas de la forme $\mathbb{A}^{r} \times \mathbb{G}_{m}^{s}$ d'après la proposition 3.1, donc on a bien $\left(i_{v, w}^{!} j_{w !} K\right)_{x_{v}} \in \mathcal{T}\left(x_{v}\right)$.

Enfin, l'isomorphisme ci-dessus donne, pour tout $j \in \mathbb{N}^{*}$,

$$
\varphi\left(\left[i_{v, w}^{!} j_{w !} K\right]\right)\left(q^{j}\right)=\operatorname{Tr}\left(F^{j *},\left(i_{v, w}^{!} j_{w !} K\right)_{x_{v}}\right)=\operatorname{Tr}\left(F^{j *}, R \Gamma_{c}\left(\left(X_{w} \cap X^{v}\right)_{\mathbb{\mathbb { F }}_{q}}, \mathbb{Q}_{\ell}(m)\right)\right) .
$$

D'après la formule des points fixes de Grothendieck-Lefschetz (SGA 4 1/2 Rapport 1.3.2) et le sous-lemme 2 ,

$$
\begin{aligned}
\operatorname{Tr}\left(F^{j *}, R \Gamma_{c}\left(\left(X_{w} \cap X^{v}\right)_{\overline{\mathbb{F}}_{q}}, \mathbb{Q}_{\ell}(m)\right)\right) & =\sum_{x \in\left(X_{w} \cap X^{v}\right)\left(\mathbb{F}_{q^{j}}\right)} \operatorname{Tr}\left(F_{x}^{*}, \mathbb{Q}_{\ell}(m)\right) \\
& =q^{-j m} \operatorname{card}\left(\left(X_{w} \cap X^{v}\right)\left(\mathbb{F}_{q^{j}}\right)\right)=q^{-j m} R_{v, w}\left(q^{j}\right) .
\end{aligned}
$$


Donc, pour tout $j \in \mathbb{N}^{*}$,

$$
\varphi\left(\left[i_{v, w}^{!} j_{w !} K\right]\right)\left(q^{j}\right)=q^{-j m} R_{v, w}\left(q^{j}\right)=\varphi([K])\left(q^{j}\right) R_{v, w}\left(q^{j}\right),
$$

ce qui implique la dernière égalité de (ii).

(iii) Le complexe $i_{v, w}^{*} I C_{\bar{X}_{w}}$ est un complexe $B$-équivariant sur $X_{v}$, donc ses faisceaux de cohomologie sont constants, et il suffit comme dans (ii) de montrer que $I C_{\bar{X}_{w}, x_{v}} \in \mathcal{T}\left(x_{v}\right)$. Ceci résulte du théorème 1.1 .

Le premier sous-lemme est prouvé par Springer dans $[\mathrm{S}]$, 3, proposition 1.

Sous-lemme 1 Soit $X$ une variété sur $\mathbb{F}_{q}$ munie d'une action de $\mathbb{G}_{m}$ qui contracte $X$ sur un point $a \in X$ (c'est-à-dire que, pour tout $\left.x \in X, \lim _{\lambda \rightarrow 0} \lambda . x=a\right)$. Soit $K$ un complexe $\ell$-adique $\mathbb{G}_{m}$-équivariant sur $X$. Alors le morphisme d'adjonction $a_{!} a^{!} K \longrightarrow K$ induit un isomorphisme

$$
a^{!} K \stackrel{\sim}{\longrightarrow} R \Gamma_{c}(X, K) .
$$

Le deuxième sous-lemme est une observation de Kazhdan et Lusztig ([KL1] lemmes A3 et A4, voir aussi [KL2] 4.6).

Sous-lemme 2 Pour tout $v \leqslant w$, pour tout $j \in \mathbb{N}^{*}$, on a

$$
R_{v, w}\left(q^{j}\right)=\operatorname{card}\left(\left(X_{w} \cap X^{v}\right)\left(\mathbb{F}_{q^{j}}\right)\right) .
$$

Démonstration du théorème 4.1. Avec les notations ci-dessus, le théorème 1.1 implique : pour tous $v, w \in W$ tels que $v \leqslant w$, on a

$$
P_{v, w}(t)=\varphi\left(\left[i_{v, w}^{*} I C_{\bar{X}_{w}}\right]\right) .
$$

Le résultat cherché résulte alors directement du théorème 2.2 et du lemme 4.2 .

\section{Références}

[BBD] A. Beilinson, J. Bernstein et P. Deligne, Analyse et topologie sur les espaces singuliers (I), Astérique 100 (1982)

[BB] A. Bialynicki-Birula, Some theorems on actions of algebraic groups, Annals of Math. 98, n³ (1973), p 480-493.

[B] F. Brenti, Lattice Paths and Kazhdan-Lusztig polynomials, Journal of the AMS, Vol. 11, n² (avril 1998), p. 229-259.

[D1] M. Demazure, Désingularisation des variétés de Schubert généralisées, Annales scientifiques de l'É.N.S $4^{e}$ série, tome 7, n¹ (1974), p 53-88. 
[D2] V. Deodhar, On some geometric aspects of Bruhat orderings. I. A finer decomposition of Bruhat cells, Invent. Math. 79 (1985), p. 499-511.

$[\mathrm{H}]$ M. Härterich, The T-equivariant cohomology of Bott-Samelson varieties, arXiv :math.AG/0412337 (2004).

[KL1] D. Kazhdan et G. Lusztig, Representations of Coxeter groups and Hecke algebras, Inventiones math. 53 (1979), p 165-184.

[KL2] D. Kazhdan et G. Luzstig, Schubert varieties and Poincaré duality, Proceedings of Symposia in Pure Mathematics 36 (1980), p 185-203.

$[\mathrm{M}] \quad$ S. Morel, Complexes pondérés sur les compactifications de Baily-Borel. Le cas des variétés de Siegel, Journal of the AMS 21 (2008), no. 1, p 23-61.

[S] T.A. Springer, A purity result for fixed point varieties in flag manifolds, Journal Fac. Sci. Univ. Tokyo 31 (1984), p 271-282. 